\title{
Evaluation of the Treatment Efficacy and Safety of Remdesivir for COVID-19: a Meta-analysis
}

\author{
Jun Tao ${ }^{1}$ (D) - Rebecca Aristotelidis ${ }^{2}$ - Alexandra Zanowick-Marr ${ }^{1} \cdot$ Laura C. Chambers $^{3}$ - James McDonald ${ }^{3}$. \\ Eleftherios E. Mylonakis ${ }^{1}$. Philip A. Chan ${ }^{1}$
}

Accepted: 1 July 2021 / Published online: 7 August 2021

(C) The Author(s), under exclusive licence to Springer Nature Switzerland AG 2021

\begin{abstract}
Remdesivir is one of few FDA-approved treatments for severe cases of Coronavirus Disease 2019 (COVID-19). To better assess its efficacy and safety, we conducted a meta-analysis to systematically identify and synthesize existing findings. We conducted a comprehensive literature search among six electronic databases and unpublished studies. Random-effects meta-analyses were performed to summarize the risk ratio (RR) and rate estimates from eligible studies. Funnel plots, the Egger test, and the trim and fill analysis were used to detect publication bias. Thirteen eligible studies were included in this meta-analysis, giving a pooled sample size of 10,002 COVID-19 hospitalized patients (5068 administered remdesivir; 4934 control). Among patients on remdesivir, we synthesized mortality $(15 \%, 95 \%$ confidence interval [CI]: 9\%, 22\%), clinical improvement $(64 \%, 95 \%$ CI: $51 \%, 78 \%)$, recovery $(70 \%, 95 \%$ CI: 57\%, 83\%), hospital discharge (74\%, 95\% CI: 60\%, 87\%), serious adverse effect (SAE) $(21 \%, 95 \%$ CI:13\%, 29\%), and Grade 3 or 4 adverse effect (AE) (30\%, 95\% CI: $12 \%, 48 \%)$. Patients on remdesivir were 17\% (RR: $0.83,95 \%$ CI: $0.65,1.06$ ) less likely to die than those within the control group. Additionally, remdesivir had favorable outcomes in terms of clinical improvement, recovery, and hospital discharge. Lastly, non-mechanically ventilated patients had better overall clinical outcomes than mechanically ventilated patients. Remdesivir shows a moderate-favorable treatment efficacy among hospitalized COVID-19 patients with disproportionate impact among non-mechanically ventilated patients; however, a substantial proportion of COVID-19 patients may suffer from SAE or Grade 3 or 4 AE during the treatment course.
\end{abstract}

Keywords Remdesivir · COVID-19 · Treatment efficacy $\cdot$ Adverse effect

\section{Background}

Coronavirus disease 2019 (COVID-19) is caused by severe acute respiratory syndrome coronavirus 2 (SARS-CoV-2) and was first reported in December 2019 in China [1]. This outbreak rapidly developed into an international crisis that is now recognized as an ongoing, global pandemic with over a hundred

This article is part of the Topical Collection on COVID-19

Jun Tao

jun_tao@brown.edu

$\triangle$ Philip A. Chan

philip_chan@brown.edu

1 Department of Medicine, Infectious Diseases, Brown University, Providence, RI, USA

2 Wellesley College, Wellesley, MA, USA

3 Rhode Island Department of Health, Providence, RI, USA million cases and millions of deaths [2]. The clinical presentation of COVID-19 varies from asymptomatic infection to critical illness with severe cases resulting in multi-organ failure that require intensive care unit (ICU) admission for mechanical ventilation, renal replacement therapy, and vasopressor support for refractory shock [1]. Further, studies on latent signs and symptoms of SARS-CoV-2 have found that $80-90 \%$ of patients present with ground-glass opacity (GGO) lesions regardless of disease severity $[3,4]$ and that infection induced immune inflammation could cause damage to the cardiovascular system $[5,6]$, heart [7], brain [8], liver [9, 10], and kidney [11]. Ultimately, these long-term effects present additional factors to consider when assessing the risk for permanent damage and reduced quality of life among COVID-19 patients.

Remdesivir (GS-5734), a viral RNA-dependent RNA polymerase (RdRp) inhibitor, can compete with native adenosine triphosphate (ATP) for incorporation into new RNA strands. This induces "chain termination" or inhibition of viral genome replication by means of premature termination of 
RNA synthesis, ultimately resulting in the suppression of virus replication [12]. Remdesivir has been demonstrated to suppress antiviral activity against a broad range of RNA viral infections, including coronavirus (SARS-CoV and Middle East respiratory syndrome coronavirus [MERS-CoV]) and filoviruses (Ebola) [13]. In a recent study, Wang and colleagues evaluated the in vitro activity of seven antiviral drugs against SARS-CoV2 and found that remdesivir was effective in controlling COVID-19 infection [14]. Given the promising results and rapidly worsening toll of the pandemic, remdesivir was approved for use on patients with severe COVID-19 under the Compassionate Use Act in January 2020.

The clinical condition of the first laboratory-confirmed COVID-19 patient significantly improved within $24 \mathrm{~h}$ of administration of remdesivir with no adverse side-effects observed [15]. Later, research confirming this initial observation was published - a study conducted by Grein et al. assessing clinical outcomes for COVID-19 patients in the USA, Europe, Canada, and Japan found that 36 of 53 (68\%) hospitalized patients who received at least one dose of remdesivir experienced clinical improvement [16]. Further, a clinical trial conducted by the National Institutes of Health demonstrated that remdesivir could significantly reduce recovery time [17]. In response to these promising results, an emergency authorization for use of remdesivir on hospitalized patients with severe COVID-19 was approved on May 1, 2020. However, the clinical efficacy of remedisvir among COVID-19 hospitalized patients is unclear.

In June 2020, large-scale distribution made remdesivir available to hospitalized COVID-19 patients with severe disease status in the USA. Of the limited randomized controlled trials (RCTs) and single-arm studies (no control arm) evaluating the effect of remdesivir on COVID-19, very few report inconsistent results [ [17-24]]. To better assess the overall effect of remdesivir on clinical outcomes for COVID-19, we conducted a meta-analysis to systematically identify trials and synthesize existing results.

\section{Methods}

\section{Literature Search and Study Selection}

In this study, we conducted a systematic literature search in accordance with the Preferred Reporting Items for Systematic Reviews and Meta-Analyses (PRISMA) protocol. We identified studies that assessed the treatment effect of remdesivir on COVID-19 patients, published from June 18th, 2020 to March 8th, 2021. Six electronic databases were searched: Pubmed (National Center for Biotechnology Information, Bethesda MD), Web of Science (Thomson Scientific Technical Support, New York), Proquest (Cambridge Information Group), Embase (Elsevier), Google Scholar, and ScienceDirect (Elsevier). We extended our search to preprint materials using medRxiv and to RCTs using remdesivir to treat COVID-19 patients at ClinicalTrials.org. Databases were searched beginning on June 18, 2020, and literature was extracted on March 8th, 2021 by JT and RA.

The search strategy was adjusted for each database using the following combination of MeSH terms and/or keywords, "Remdesivir OR GS-5734" AND "COVID-19 OR SARS-CoV-2". The full PubMed search strategy is described in the appendix.

\section{Study Criteria and Selection}

We determined study eligibility using the following inclusion criteria: (1) was an RCT, cohort study, chart review, or single arm prospective study; (2) assessed the treatment effect of remdesivir; (3) used COVID-19 patients as the study population; (4) measured clinical improvement, hospital discharge, recovery, mortality, or adverse events; and (5) provided sufficient information to calculate effect size (ES) estimates.

Studies identified in the literature search were imported into EndNote 8.0 (Thomson Reuters Corporation, New York). After removing duplicates, we conducted title screening and excluded irrelevant articles. We reviewed the abstracts of the remaining articles, and only studies that mentioned the use of remdesivir on COVID-19 patients were chosen for fulltext screening. JT and RA conducted the screening and selection process independently.

\section{Data Extraction}

For eligible studies, we used a standardized approach to extract the following information: first author's name, year of publication, study period, study country, study design, sample size, study population/eligibility criteria, remdesivir dose duration, method of drug delivery, control group duration, and outcomes of interests. Outcomes of interest included clinical improvement ( 2 points increase on 6-point scale), recovery, all-cause mortality, serious adverse events (SAE), and grade 3 or 4 adverse events (AE). Recovery was defined as an improvement to one of two outcomes: hospital discharge or removal from supplementary oxygen $[18,19,23]$. Adverse events were defined as grade 3 and 4 adverse events and serious adverse events. We extracted the total number of patients and number of patients with outcomes of interest in each group. JT and RA extracted information indecently and reached agreement after the data extraction.

\section{Assessment of Risk of Bias}

We used the Cochrane Collaboration's tool to assess risk of bias in the meta-analysis (http://handbook-5-1.cochrane.org/). We assessed risk of bias in different domains, including selection bias (i.e., random sequence generation and allocation 
concealment), performance bias (blinding of participants and personnel), detection bias (blinding of outcome assessment), attrition bias (incomplete outcome data), and reporting bias (selective reporting). JT and RA performed the risk of bias assessment and achieved agreement.

\section{Statistical Methods}

To account for variation in studies' reported effect sizes, we calculated and synthesized crude risk ratios (RRs) in our metaanalysis. We used random-effects meta-analyses using inverse variance weights to pool RRs among eligible studies. The natural logarithm of RRs and 95\% confidence intervals (CIs) were used for synthesis and then transformed back for ease of interpretation. For the percentage of clinical improvement, recovery, hospital discharge, death, $\mathrm{SAE}$, and grade 3 or 4 $\mathrm{AE}$, percentages and standard error were used for synthesis. Forest plots were used to display the synthesized results.

$\mathrm{Q}$ statistic and $\mathrm{I}^{2}$ statistic were used to assess heterogeneity across eligible studies. As the Q statistic is underpowered to detect true heterogeneity with a limited number of eligible studies. We used a $10 \%$ significance level for the Q statistics to increase the power to detect heterogeneity among the 13 eligible studies. Lastly, funnel plots, the Egger test, and the trim and fill analysis were used to detect publication bias. All statistical analyses were performed in Stata 16.0 (Stata Corporation, College Station, TX) by JT.

\section{Results}

\section{Results of Literature Search}

Figure 1 shows the process of our study selection. The initial searches in six individual electronic databases yielded 549 records, of which 95 were duplicates, leaving 487 records for title and abstract screening. Thirty-two publications were identified for full-text screening. Among these publication, 13 studies assessed the treatment effect of remdesivir among hospitalized COVID-19 patients and were included in our meta-
Fig. 1 The process of the study selection

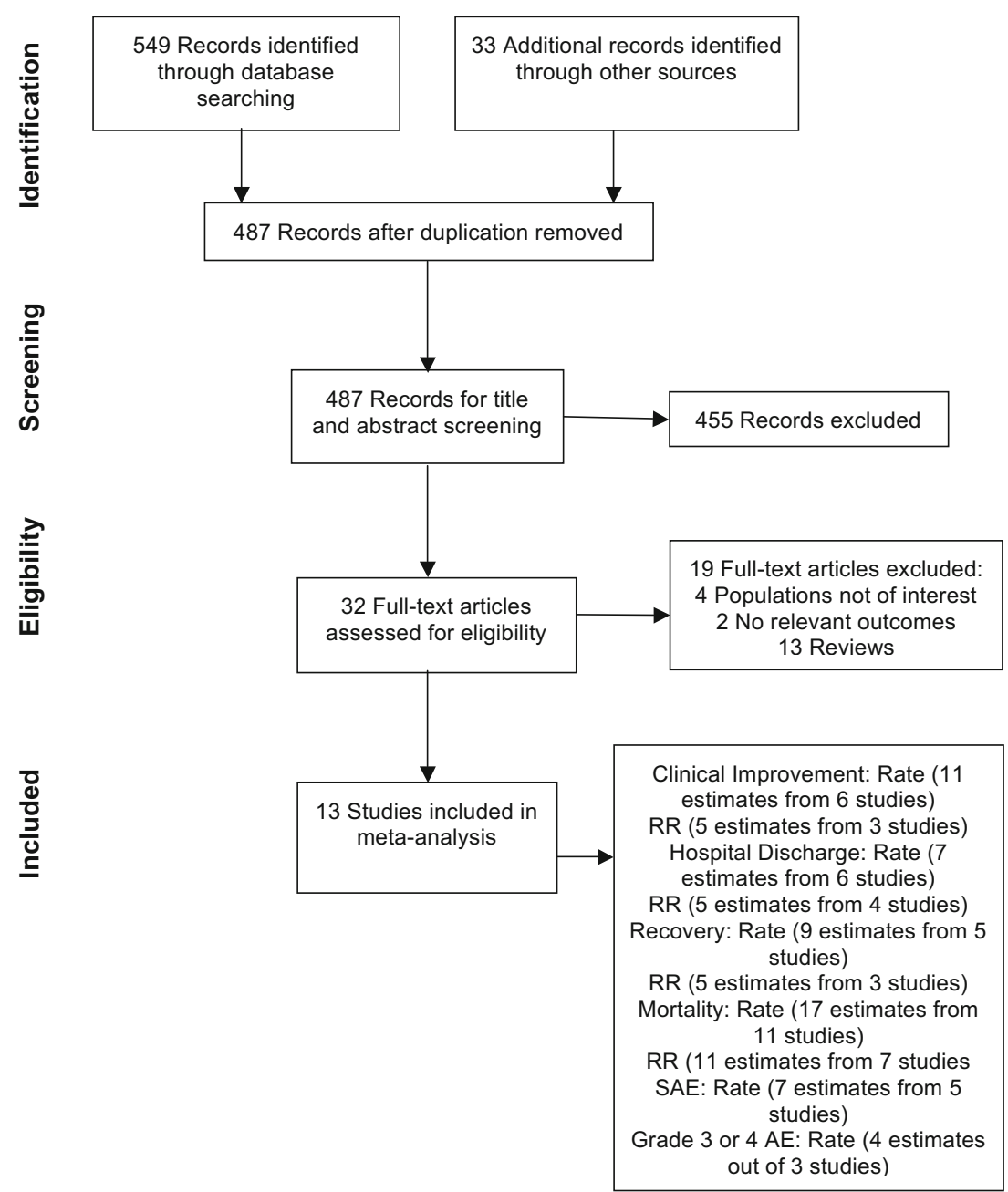


analysis. There were six RCTs, three cohort studies, three single-arm prospective studies, and one chart review.

\section{Description of Studies}

Table 1 describes the characteristics of studies included in this meta-analysis. Overall, we identified 5068 COVID-19 patients who received remdesivir and 4934 COVID-19 patients who served as controls during their hospital stay within the 13 eligible studies [16-28]. Of the 10,002 COVID-19 patients in this analysis, $70.7 \%$ were male. Most patients were over 55 years old. All participants were hospitalized due to COVID-19 and, for 11 of 13 eligible studies, all participants fell within one of the following categories: (1) had an oxygen saturation (SaO2/SPO2) of $94 \%$ or less while breathing ambient air, (2) needed supplemental oxygen, (3) needed mechanical ventilation. Among the two additional studies, one excluded patients on mechanical ventilation and extracorporeal membrane oxygenation (ECMO) [22], while the other enrolled patients with a $\mathrm{SaO} 2 / \mathrm{SPO} 2>94 \%$ [19]. Although the study by Goldman et al. was an RCT, they did not incorporate a control group. Consequently, we treated this study as a prospective cohort study. In Kalil et al.'s study, the group of COVID-19 patients who solely received remdesivir was extracted, and the study was treated as a single-arm prospective cohort study [27].

\section{Assessment of Risk Bias}

Table 2 presents the risk bias of eligible studies. RCT studies had a low risk of selection bias, as these studies all provided detailed information about randomization [17-19, 22, 25, 27]. The rest of included studies were either single-arm prospective studies, chart reviews, or cohort studies. Randomization and group allocation were not part of these study designs. We put "Not applicable" to these studies. Three studies used double-blinded study designs [17, 18, 27], and were assessed as "low-risk" for detection bias. We assigned "Not applicable" to the rest of studies due to the unblinded study design. A "low-risk" were assigned to all studies, except for Lee et al's study [21]. Lee et al extracted data from electronic medical records and did not report detail information about incomplete outcomes. Hence, we put an "unknown risk" for this study. For reporting bias, a "low-risk" was assigned to RCT studies $[17-19,22,25,27]$. All these RCT studies provided detailed results of outcomes of interests in supplementary. Other studies were assigned an "unknown risk" due to the lack of data to assess the potential reporting bias.

\section{Effect of Remdesivir on Mortality}

The mortality rate at $28 \pm 2$ days of hospitalized patients on remdesivir varied from 1 to $56 \%$ in 17 estimates from 10 studies (Fig. 2a). Several studies contributed two estimates of mortality, as heterogeneous effects were observed across different study populations (non-mechanical ventilated vs. mechanically ventilated patients) and varied regimens (5 vs. 10 days course). The pooled mortality rate was $15 \%$ (95\% CI: $9 \%, 22 \%$ ) among patients on remdesivir. When stratified by ventilation status, a $29 \%$ (95\% CI: $19 \%, 39 \%)$ mortality rate was observed among mechanically ventilated patients, and a $6 \%$ (95\% CI: 3\%, 9\%) mortality rate was observed among those without mechanical ventilation $(\mathrm{P}<0.001)$. Seven studies had a control group and reported the number of deaths in each group. The pooled RR of remdesivir on mortality was 0.83 (95\% CI: $0.65,1.06$ ) (Fig. 2b), suggesting that patients on remdesivir were $17 \%$ less likely to die when compared to COVID-19 patients in the control group.

When stratified by ventilation status, mechanically ventilated patients were $16 \%$ (RR: $0.84,95 \%$ CI: $0.52,1.35$ ) less likely to die, while non-ventilated patients were $20 \%$ (RR: $0.80,95 \%$ CI: $0.62,1.03)$ less likely to die at $28 \pm 2$ days. No significant differences between mechanical and nonmechanical groups were observed $(\mathrm{P}=0.98)$. The $\mathrm{Q}$ statistic rejected the null hypothesis that true heterogeneity was due to chance (chi-square: 20.05 , degree of freedom $=9, \mathrm{P}=0.02$ ) at a $10 \%$ significance level. The $\mathrm{I}^{2}$ statistic indicated that $60.2 \%$ of the variance could be explained by true heterogeneity.

\section{Effect of Remdesivir on Clinical Improvement}

In this meta-analysis, six studies reported 11 estimates of clinical improvement at either $14 \pm 1$ or 28 days among hospitalized patients on remdesivir (Fig. 3a). The clinical improvement rates varied from 16 to $89 \%$. The overall pooled clinical improvement rate was $64 \%$ (95\% CI: 51\%, 78\%). When stratified by period, the pooled clinical improvement rates at day $14 \pm 1$ and $28 \pm 1$ were $52 \%$ (95\% CI: $35 \%, 69 \%$ ) and $79 \%$ (95\% CI: 66\%, 93\%), respectively. In the mechanical ventilated group, the clinical improvement rate was 36\% (95\% CI: $0.14,0.58)$. A higher clinical improvement rate of $74 \%$ (95\% CI: $65 \%, 84 \%$ ) was observed in the non-mechanical ventilated group. The overall pooled RR of remdesivir on clinical improvement was $1.12(95 \%$ CI 1.05, 1.19) among three studies having a control group (Fig 3b.). Only one study reported clinical improvement in the mechanical ventilated group. Among the non-mechanical ventilated group, patients on remdesivir were $12 \%$ (RR 1.12, 95\% CI 1.05, 1.19) more likely to achieve clinical improvement than those in the control group. As the $\mathrm{Q}$ statistic was $4.13(\mathrm{P}=0.39$, degree of freedom 4 ), the true heterogeneity may be due to chance. The true heterogeneity could explain $23.7 \%$ of the variance.

\section{Effect of Remdesivir on Recovery}

Five studies contributed nine recovery rates at either $14 \pm 1$ or 28 days with a range of $37 \%$ to $93 \%$ among hospitalized 


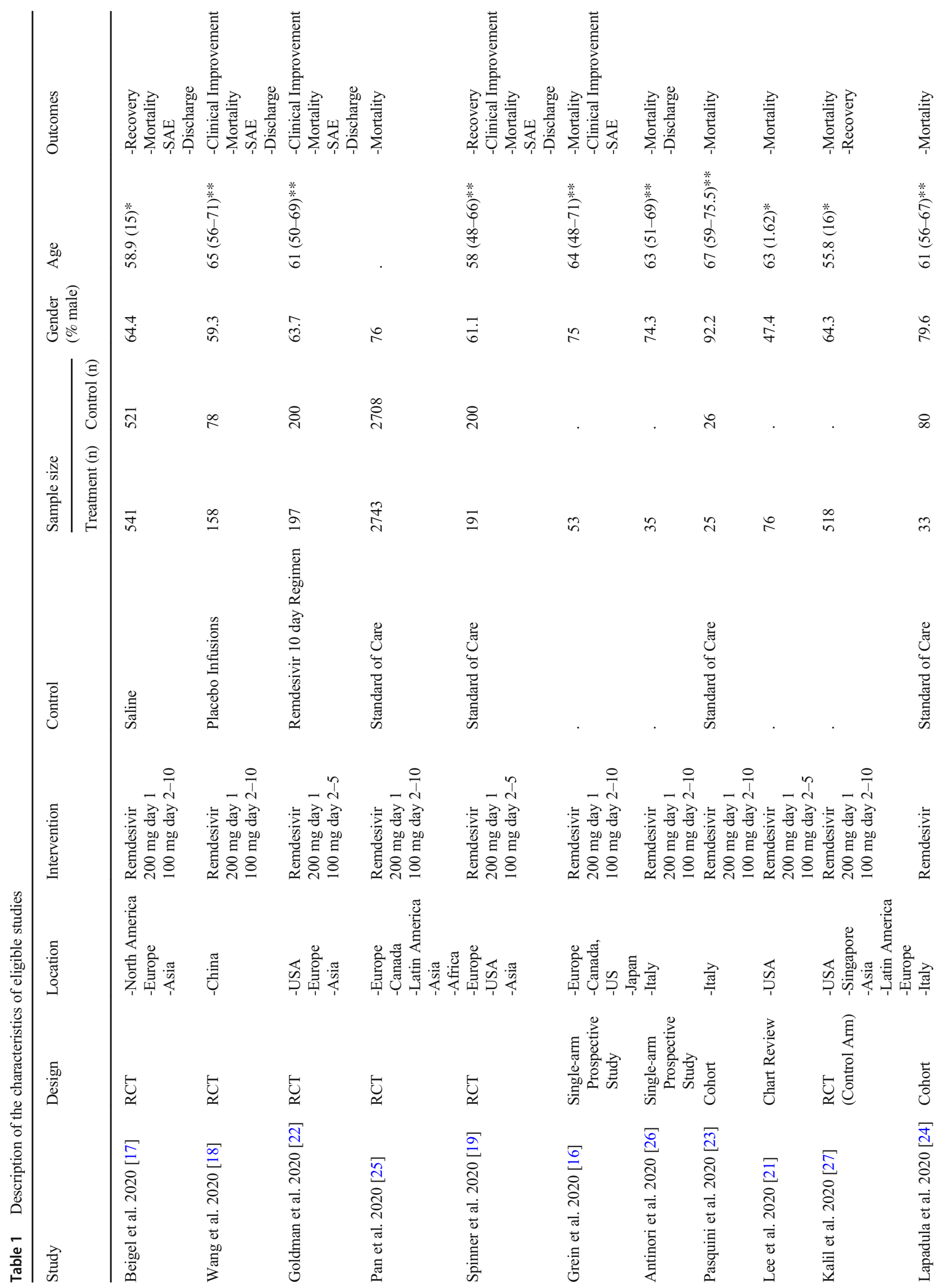


patients receiving remdesivir treatment (Fig. 4a). The overall recovery rate was $70 \%$ (95\% CI: $57 \%, 83 \%$ ). When stratified by period, the pooled recovery rates at day $14 \pm 1$ and $28 \pm 1$ were $60 \%$ (95\% CI: $41 \%, 79 \%)$ and $77 \%$ (95\% CI: $61 \%$, $93 \%)$, respectively. In the non-mechanical ventilated group, $77 \%$ (95\% CI: $57 \%, 83 \%$ ) of patients on remdesivir were recovered during the study period, while $43 \%$ (95\% CI: $33 \%, 54 \%$ ) of patients were recovered in the mechanical ventilated group. Three studies reported five estimates of RR of remdesivir on recovery. The pooled RR was 1.09 (95\% CI $1.04,1.13$ ) (Fig. 4b). Only one study reported a recovery rate among mechanically ventilated patients [18]. Nonmechanically ventilated patients receiving remdesivir were $9 \%(95 \%$ CI $1.04,1.13)$ more likely to achieve recovery than their counterparts without remdesivir. The $\mathrm{Q}$ statistic indicates the true heterogeneity across studies may be due to chance (chi-square 1.57, degree of freedom $4, \mathrm{P}=0.81$ ).

\section{Effect of Remdesivir on Hospital Discharge}

There were seven estimates of the hospital discharge rate at 28 days from five studies. These estimates ranged from 33 to 90\% among COVID-19 patients on remdesivir treatment (Fig. 5a). The pooled hospital discharge rate was 74\% (95\% CI: $60 \%, 87 \%)$. The hospital discharge rates were $60 \%(95 \%$ CI: $9 \%, 100 \%)$ and $81 \%(95 \%$ CI: $67 \%, 95 \%)$ in mechanical and non-mechanically ventilated groups, respectively. The pooled RR of remdesivir on hospital discharge based on five RRs from four studies was 1.12 (95\% CI 1.06, 1.19) (Fig. 5b). From results reported by Lapadula et al., mechanically ventilated COVID-19 patients on remdesivir were 39\% (RR 1.39, $95 \%$ CI 1.10, 1.74) more likely to be discharged than their counterparts on the standard of care [24]. The synthesized RR among non-mechanical ventilated patients was 1.08 (95\% CI $1.06,1.19)$. The true heterogeneity across studies could be due to chance (chi-square 6.50, degree of freedom $=4, \mathrm{P}=0.16$ ) at a $10 \%$ significance level. The true heterogeneity could explain $26.4 \%$ of the variance.

\section{SAE and Grade 3 or 4 AE Among COVID-19 Patients on Remdesivir}

Seven estimates of SAE rates from five studies (Fig. 6a) and four estimates of Grade 3 or $4 \mathrm{AE}$ from three studies (Fig. 6b) were found. The pooled SAE rate and Grade 3 or 4 rate were $21 \%$ (95\% CI: $13 \%, 29 \%)$ and 30\% (95\% CI: $12 \%, 48 \%$ ), respectively. Mechanically ventilated COVID-19 patients on remdesivir were more likely to have an SAE (35\%) when compared to non-mechanically ventilated patients (20\%) (Fig. 6a). The true heterogeneity across studies for both SAE and Grade 3 or $4 \mathrm{AE}$ were not due to chance, as Q statistics rejected the null hypothesis at a $10 \%$ significance level. 
Table 2 Summary of risk of bias among eligible studies in this meta-analysis

\begin{tabular}{|c|c|c|c|c|c|c|}
\hline \multirow[t]{2}{*}{ Study } & \multicolumn{2}{|l|}{ Selection bias } & \multirow{2}{*}{$\begin{array}{l}\text { Performance bias } \\
\text { Blinding of participants } \\
\text { and personnel }\end{array}$} & \multirow{2}{*}{$\begin{array}{l}\text { Detection bias } \\
\text { Blinding of outcome } \\
\text { assessment }\end{array}$} & \multirow{2}{*}{$\begin{array}{l}\text { Attrition bias } \\
\text { Incomplete } \\
\text { outcome data }\end{array}$} & \multirow{2}{*}{$\begin{array}{l}\text { Reporting bias } \\
\text { Selective reporting }\end{array}$} \\
\hline & $\begin{array}{l}\text { Random sequence } \\
\text { generation }\end{array}$ & $\begin{array}{l}\text { Allocation } \\
\text { concealment }\end{array}$ & & & & \\
\hline Beigel et al. 2020 [17] & Low risk & Low risk & Low risk & Low risk & Low risk & Low risk \\
\hline Wang et al. 2020 [18] & Low risk & Low risk & Low risk & Low risk & Low risk & Low risk \\
\hline Goldman et al.2020 [22] & Low risk & Low risk & NA & NA & Low risk & Low risk \\
\hline Pan et al. 2020 [25] & Low risk & Low risk & NA & NA & Low risk & Low risk \\
\hline Spinner et al. 2020 [19] & Low risk & Low risk & NA & NA & Low risk & Low risk \\
\hline Grein et al. 2020 [16] & NA & NA & NA & NA & Low risk & Unknown risk \\
\hline Antinori et al. 2020 [26] & NA & NA & NA & NA & Low risk & Unknown risk \\
\hline Pasquini et al. 2020 [23] & NA & NA & NA & NA & Low risk & Unknown risk \\
\hline Lee et al. 2020 [21] & NA & NA & NA & NA & Unknown risk & High risk \\
\hline Kalil et al. 2020 [27] & Low risk & Low risk & Low risk & Low risk & Low risk & Low risk \\
\hline Lapadula et al. 2020 [24] & NA & NA & NA & NA & Low risk & Unknown risk \\
\hline Rivera et al. 2020 [20] & NA & NA & NA & NA & Low risk & Unknown risk \\
\hline Falcão et al. 2021 [28] & NA & NA & NA & NA & Low risk & Unknown risk \\
\hline
\end{tabular}

NA not applicable

\section{Publication Bias}

For all outcomes of interest, funnel plots of RRs were symmetric (Supplementary Fig 1a-1d) and Egger tests indicated Pvalues greater than 0.05 . The trim and fill test did not trim or fill any studies included within this meta-analysis. As a consequence, we concluded that there was no significant publication bias for RRs of the outcomes of interest.

For rates of mortality, clinical improvement, recovery, hospital discharge, SAE, Grade 3 or $4 \mathrm{AE}$, funnel plots tended to be symmetric, and several results were out of the pseudo 95\% CI (Supplementary Fig 2a-2f). The Egger test of mortality and

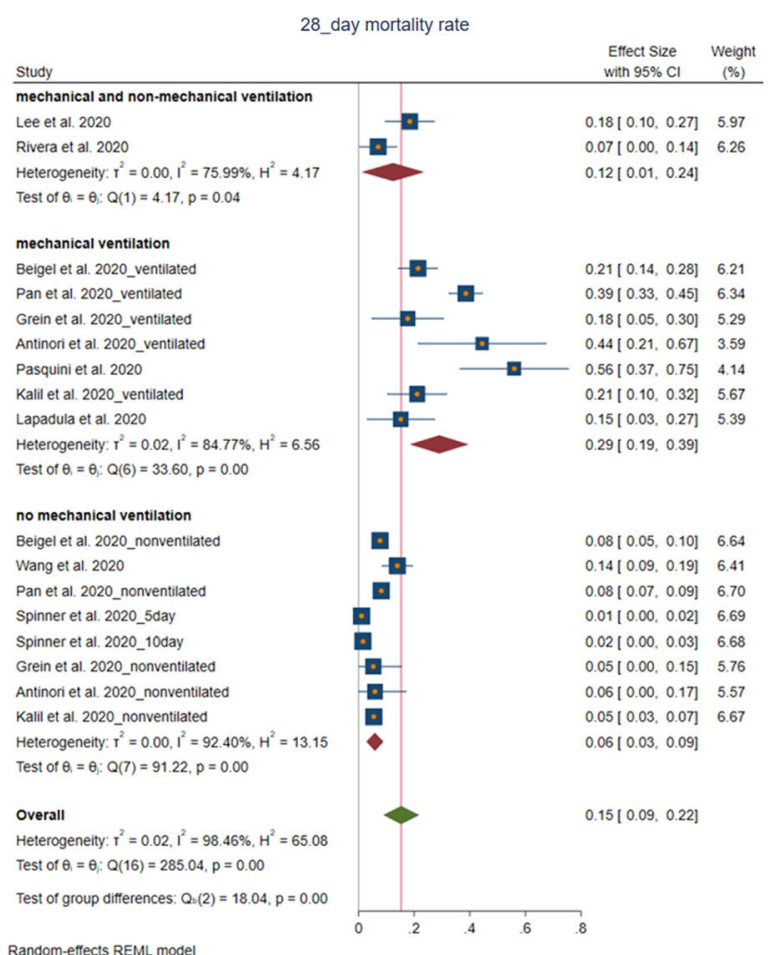

Random-effects REML model

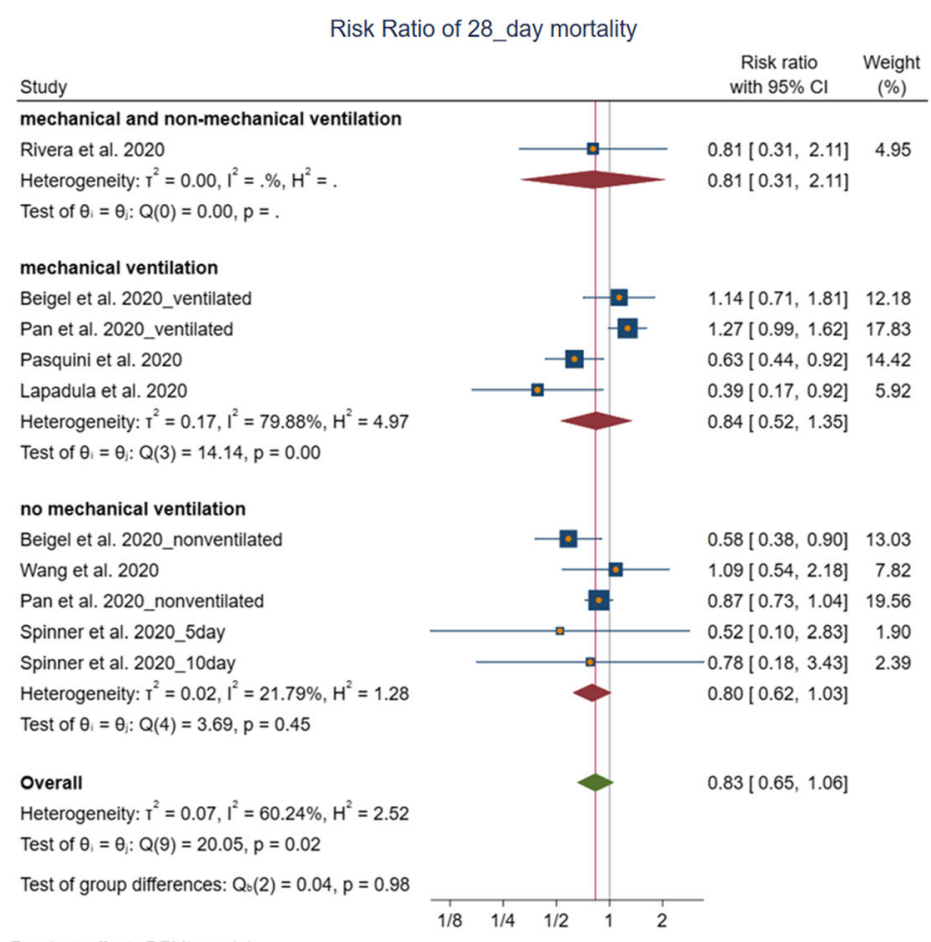

Random-effects REML model

Fig. 2 The 28-day mortality rate of hospitalized patients on remdesivir in 17 estimates from 10 studies 


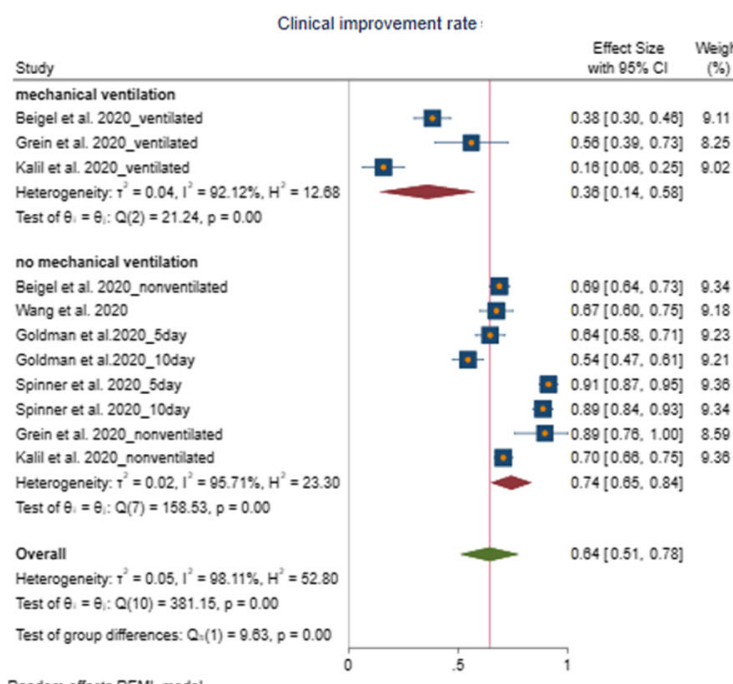

A
Risk ratio of clinical improvement

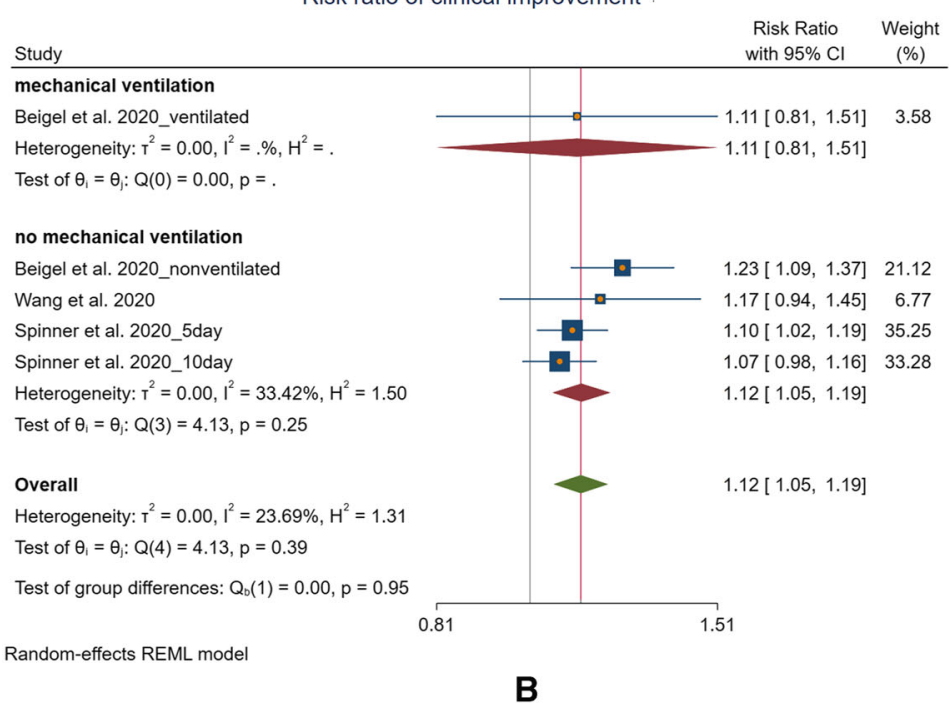

Fig. 3 Six studies reported 11 estimates of clinical improvement among hospitalized patients on remdesivir

recovery rates rejected the null hypothesis that no small study exits $(\mathrm{P}<0.05)$. Thus, although the trim and fill analysis did not yield any trim or fill, publication bias may still exist for mortality and recovery rates. The Egger test of clinical improvement, hospital discharge, SAE, and Grade 3 or $4 \mathrm{AE}$ did not reject the null hypothesis $(\mathrm{P}>0.05)$, and the trim and fill test did not yield any trim or fill for these outcomes. Therefore, publication bias was unlikely to exist for these variables.

\section{Discussion}

This study is one of a few meta-analyses to synthesize multiple clinical outcomes (e.g., mortality, clinical improvement, recovery, and hospital discharge) and safety (SAE and Grade 3 or 4 AE) of remdesivir from varied study designs. From our results, remdesivir was found to have a moderate-favorable effect on hospitalized COVID-19 patients with significant, and favorable effects on clinical improvement, recovery, and hospital discharge. However, we did not detect a significant effect of remdesivir on mortality. Finally, our results suggest that $20-30 \%$ of patients on remdesivir may experience an SAE or Grade 3 or 4 AE.

Generally, the administration of remdesivir and the assessment of clinical outcomes were comparable across studies. Because eligible studies predominantly adopted the ordinal score to assess clinical outcomes, assessment of these factors was consistent across studies and the potential influence of

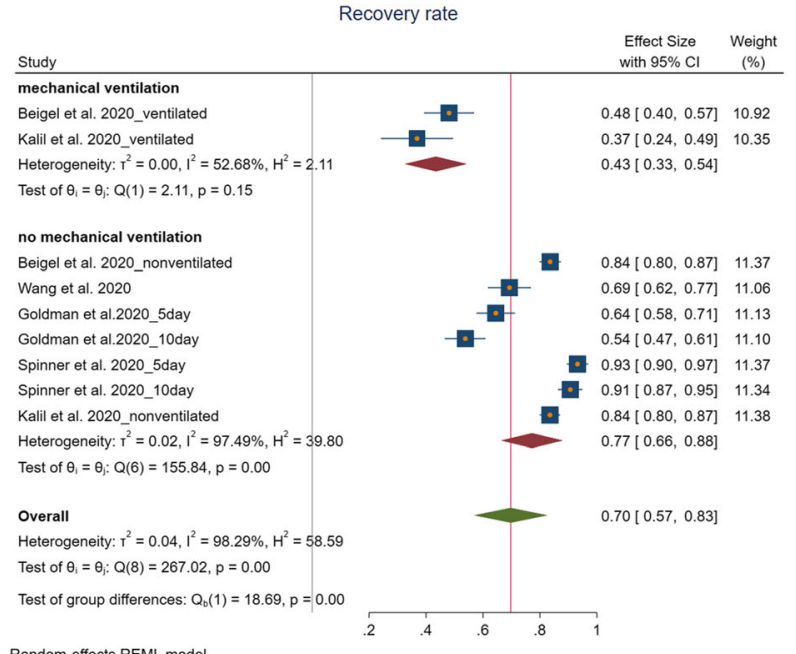

A

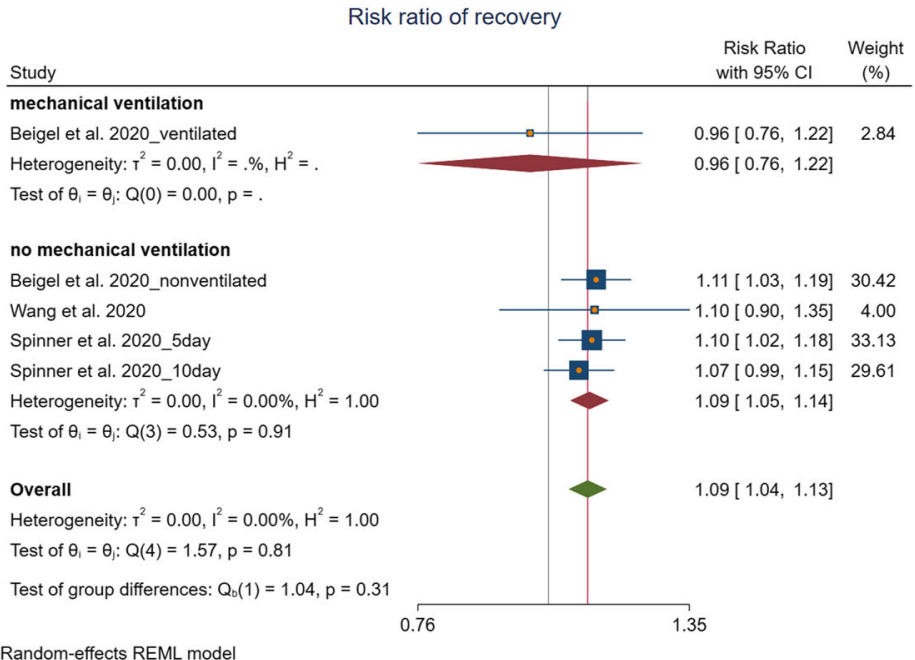

Fig. 4 Five studies contributed nine recovery rates among hospitalized patients receiving remdesivir treatment 


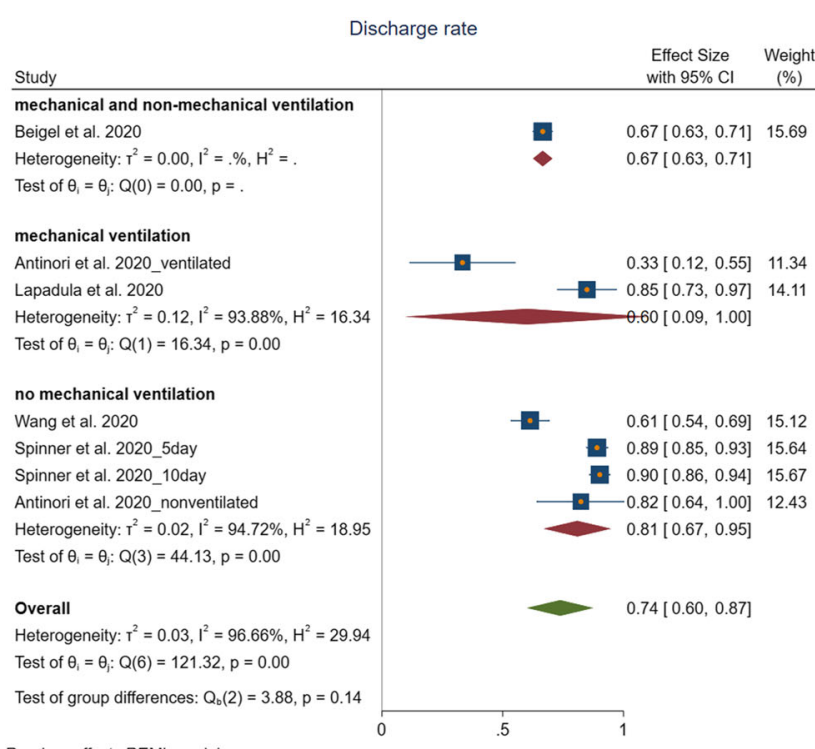

A

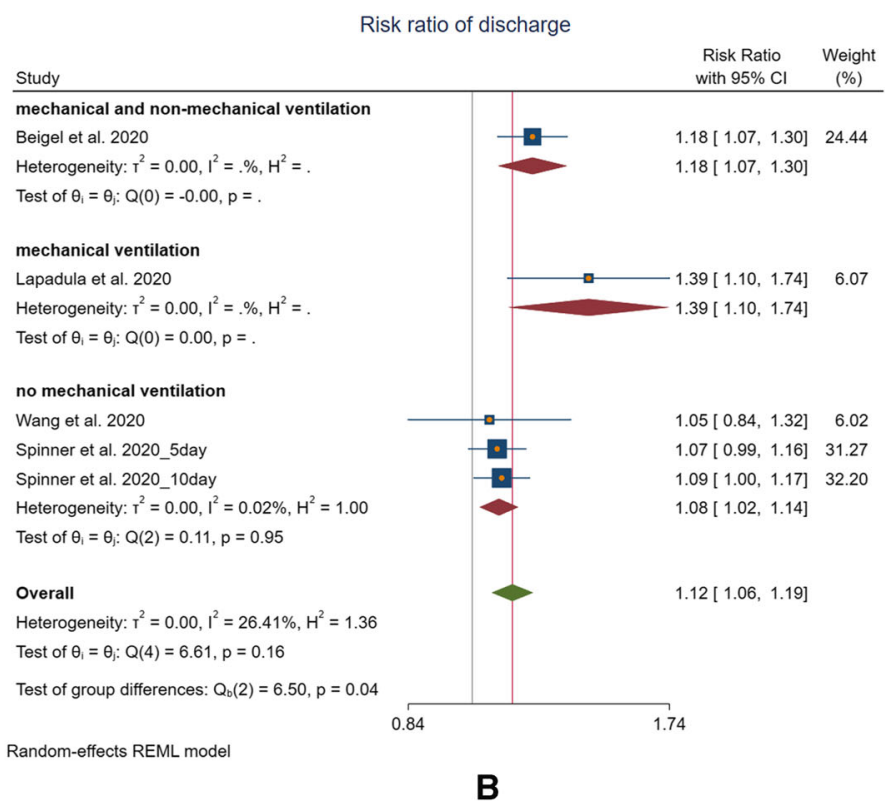

Risk ratio of discharge

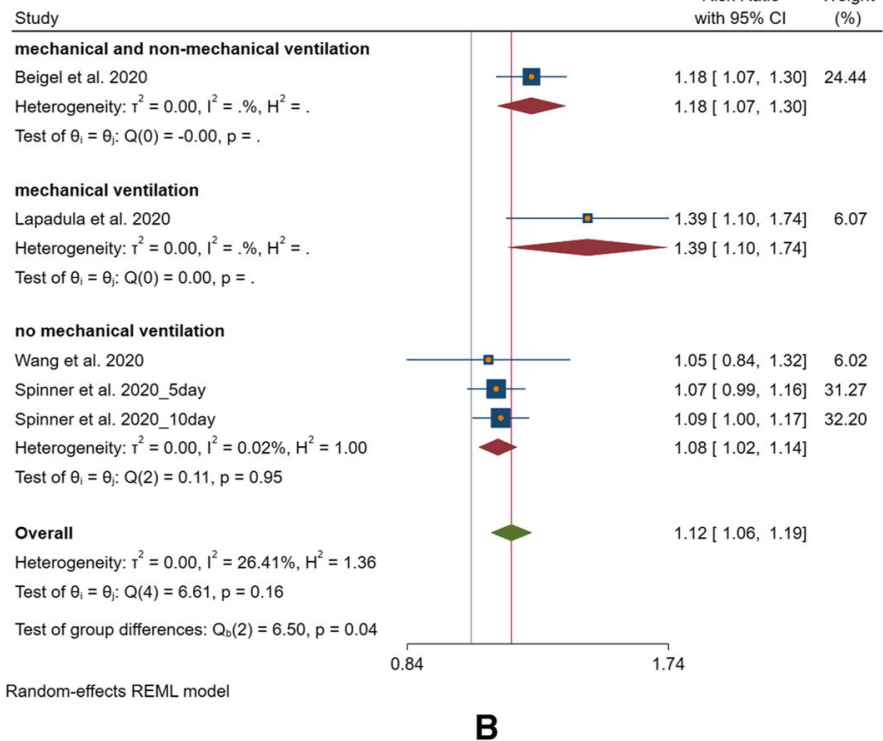

Fig. 5 Seven estimates of the hospital discharge rate at 28 days from five studies

reporting bias is limited. Additionally, most studies used the recommended 10-day course of remdesivir. Only Goldman et al. and Spinner et al. included patient groups who received a 5-day course of treatment $[19,22]$. Even so, results of interest were not significantly different between different treatment regimens.

Our heterogeneity assessment suggested that the majority of variances among rates of outcomes of interest were attributable to true heterogeneity. This, however, may additionally be caused by the variation in study populations introduced by case severity or study location [16-28]. Although most hospitalized COVID-19 patients were male and in their 50s across studies, the disease severity varied significantly. Eligible studies could include any combination of ventilated and nonventilated patients [16-28]. Further, patients with severe clinical status were at higher risk of death and lower risk of clinical improvement, recovery, and hospital discharge, consequently resulting in a trend towards worse clinical outcomes within studies assessing this population. When stratified by ventilation status, results still indicated significant heterogeneity. As previously mentioned, study location is an additional factor that may contribute to this finding as pandemic status, healthcare resources, and treatment guidelines can drastically vary by study location. Several eligible studies included

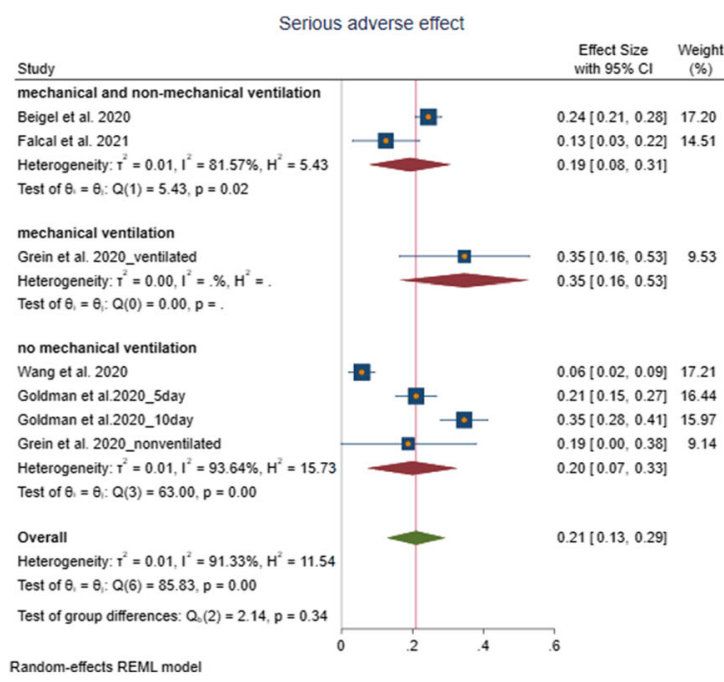

A

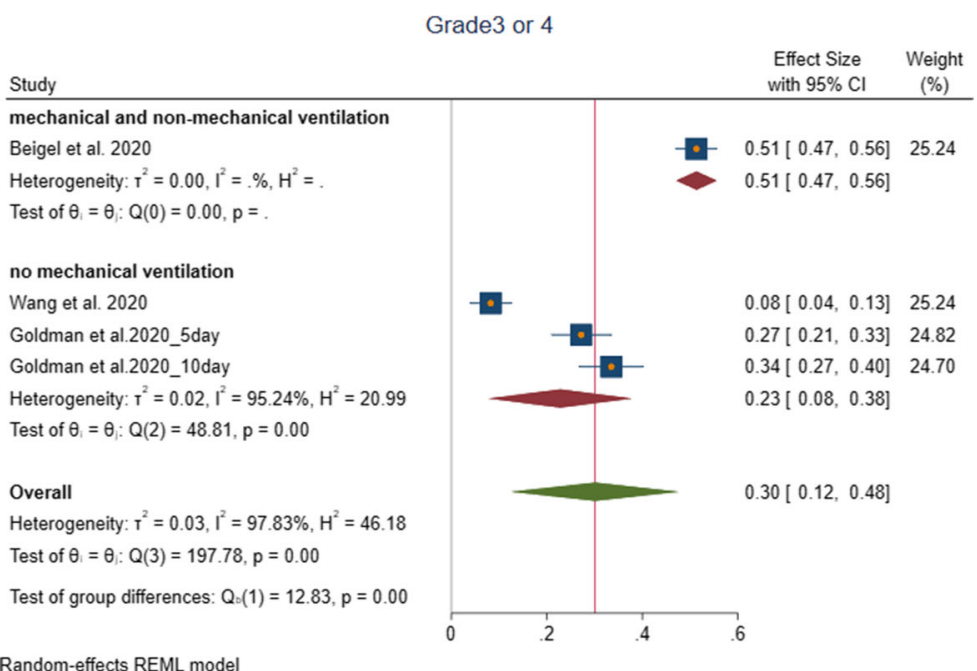

B

Fig. 6 Seven estimates of SAE rates from five studies and four estimates of Grade 3 or 4 AE from three studies 
within the meta-analysis were multi-site research studies spanning multiple countries [16, 17, 19, 22]. Other eligible studies were either single or multi-site studies conducted within the same country [18, 23, 24]. Although significant variance in rates was observed among outcomes of interest in this metaanalysis, the pooled results serve as a valuable summary of clinical outcomes among hospitalized COVID-19 patients worldwide.

When it comes to RRs of clinical outcomes, heterogeneity assessment indicated that the majority of variances of RRs of outcomes of interests were due to chance, except for RR of mortality. The majority of studies reporting RRs were either RCTs or cohort studies, both of which are known to characteristically have more rigorous operational procedures and clinical guidelines. Naturally, RRs serve as a comparison between intervention and control groups and, as a result, are less likely to be influenced by varied population and/or locationspecific risk factors. Though we were able to identify four studies assessing RR of mortality among mechanically ventilated patients, two cohort studies in Italy indicated a favorable effect of remdesivir on mortality [23, 24], while the other two studies with multiple study sites suggested a worse survival among ventilated patients on remdesivir $[17,25]$ As a consequence, more studies are needed to explore remdesivir's effect on mortality among ventilated COVID-19 patients.

Our meta-analysis has a few strengths and limitations. This meta-analysis is a comprehensive assessment of the effect of remdesivir on multiple clinical outcomes (e.g., clinical improvement, hospital discharge, and mortality), while other published meta-analyses only synthesized mortality and/or adverse effect from a few studies [29-32]. In addition, we included cohort studies, chart reviews, and single-arm prospective studies, which could reflect the effectiveness of remdesivir in realworld clinical settings than those only included RCT studies. [29-32] There are several limitations in this study. We observed significant true heterogeneity across studies, even when we stratified by the severe of disease status. This could lead to biased synthesized results. Reporting biases could exist among non-RCT studies, as these studies usually had less restricted protocol of results reporting.

In conclusion, our study serves as a comprehensive metaanalysis to evaluate the effect of remdesivir on multiple clinical outcomes among hospitalized COVID-19 patients. Our results suggest that remdesivir has a moderate-favorable effect on mortality, clinical improvement, recovery, and hospital discharge among non-mechanically ventilated patients as opposed to mechanically ventilated patients. Overall, results from the meta-analysis suggest that remdesivir is beneficial for hospitalized COVID-19 patients and may be potentially impactful as a treatment strategy in non-mechanically ventilated patient populations.

Abbreviations AE, Adverse events; CI, Confidence interval; ECMO, Extracorporeal membrane oxygenation; ES, Effect size; GGO, Groundglass opacity; ICU, Intensive care unit; PRISMA, Preferred Reporting Items for Systematic Reviews and Meta-Analyses; RCT, Randomized controlled trial; RdRp, RNA-dependent RNA-polymerase; RR, Risk ratio; SAE, Serious adverse events; SARS-CoV-2, Severe Acute Respiratory Syndrome Coronavirus 2 (SARS-CoV-2); WHO, World Health Organization

Supplementary Information The online version contains supplementary material available at https://doi.org/10.1007/s42399-021-01014-y.

Author Contribution JT and RA conducted the literature search, study screening and selection, data extraction, and assessment of the risk of bias. JT performed the statistical analysis and draft this manuscript. AZ, LC, JM, EEM, and PAC facilitated this study, provided feedback, and modified this manuscript.

Data Availability All data generated or analyzed during this study are included in this published article and its supplementary information files.

Code Availability Stata code will be available if requested.

\section{Declarations}

Ethics Approval This retrospective chart review study involving human participants was in accordance with the ethical standards of the institutional and national research committee and with the 1964 Helsinki Declaration and its later amendments or comparable ethical standards. The Human Investigation Committee (IRB) of The Miriam Hospital approved this study.

Consent to Participate Not applicable.

Consent for Publication Not applicable.

Competing Interests The authors declare no competing interests.

\section{Appendix}

The search strategy for PubMed is listed below:

(1) Remdesivir strings: (Remdesivir [Supplementary Concept] OR GS-5734 OR “GS 5734" OR "GS-441524 triphosphate")

(2) Coronavirus strings: (COVID-19 [Supplementary Concept] OR SARS-CoV-2 [Supplementary Concept] AND "2019 novel coronavirus disease" OR "COVID-19 pandemic" OR "SARS-CoV-2 infection" OR "COVID-19 virus disease" OR "2019 novel coronavirus infection" OR "2019-nCoV infection" OR "coronavirus disease 2019" OR "severe 
acute respiratory coronavirus syndrome 2" OR "coronavirus disease-19" OR "2019-nCoV disease" OR "COVID-19 virus infection")

(3) Published year: ("2019"[PDAT] : "2020"[PDAT])

(4) The strategy was $1 \& 2 \& 3$

\section{References}

1. Huang C, Wang Y, Li X, Ren L, Zhao J, Hu Y, et al. Clinical features of patients infected with 2019 novel coronavirus in Wuhan, China. Lancet. :102232020, 497-506.

2. WHO COVID-19 Dashboard. Geneva: World Health Organization. 2020. https://covid19.who.int/. Accessed March, 152021.

3. Meng H, Xiong R, He R, Lin W, Hao B, Zhang L et al. CT imaging and clinical course of asymptomatic cases with COVID-19 pneumonia at admission in Wuhan, China. J Infect. 2020:81(1):e33-e39. https://doi.org/10.1016/j.jinf.2020.04.004

4. Zhao W, Zhong Z, Xie X, Yu Q, Liu J. Relation between chest CT findings and clinical conditions of Coronavirus Disease (COVID19) Pneumonia: A Multicenter Study. AJR Am J Roentgenol. 2020;214(5):1072-7. https://doi.org/10.2214/ajr.20.22976.

5. Aggarwal G, Cheruiyot I, Aggarwal S, Wong J, Lippi G, Lavie CJ, et al. Association of Cardiovascular Disease With Coronavirus Disease 2019 (COVID-19) severity: a meta-analysis. Curr Probl Cardiol. Curr Probl Cardiol. 2020;45(8):100617. https://doi.org/10.1016/j. cpcardiol.2020.100617.

6. Ferrari F. COVID-19: Updated data and its relation to the cardiovascular system. Arq Bras Cardiol. 2020;114(5):823-826. https:// doi.org/10.36660/abc.20200215

7. Akhmerov A, Marban E. COVID-19 and the heart. Circ Res. 2020;126(10):1443-55. https://doi.org/10.1161/circresaha.120. 317055.

8. Li YC, Bai WZ, Hashikawa T. The neuroinvasive potential of SARS-CoV2 may play a role in the respiratory failure of COVID19 patients. J Med Virol. 2020;92:552-5. https://doi.org/10.1002/ jmv.25728.

9. Feng G, Zheng KI, Yan QQ, Rios RS, Targher G, Byrne CD, et al. COVID-19 and liver dysfunction: current insights and emergent therapeutic strategies. J Clin Trans1 Hepatol. 2020;8(1):18-24. https://doi.org/10.14218/jcth.2020.00018.

10. Zhang C, Shi L, Wang FS. Liver injury in COVID-19: management and challenges. Lancet Gastroenterol Hepatol. 2020;5(5):428-30. https://doi.org/10.1016/s2468-1253(20)30057-1.

11. Sise ME, Baggett MV, Shepard JO, Stevens JS, Rhee EP. Case 172020: A 68-year-old man with Covid-19 and acute kidney injury. $\mathrm{N}$ Eng1 J Med. 2020;382:2147-56. https://doi.org/10.1056/ NEJMcpc2002418.

12. Eastman RT, Roth JS, Brimacombe KR, Simeonov A, Shen M, Patnaik $\mathrm{S}$ et al. Remdesivir: a review of its discovery and development leading to human clinical trials for treatment of COVID-19. ACS Cent Sci. 2020;6(5):672-683. https://doi.org/10.1021/ acscentsci.0c00489.

13. Liang C, Tian L, Liu Y, Hui N, Qiao G, Li H, et al. A promising antiviral candidate drug for the COVID-19 pandemic: a mini-review of remdesivir. Eur J Med Chem. 2020;201:112527. https:// doi.org/10.1016/j.ejmech.2020.112527.

14. Wang M, Cao R, Zhang L, Yang X, Liu J, Xu M, et al. Remdesivir and chloroquine effectively inhibit the recently emerged novel coronavirus (2019-nCoV) in vitro. Cell Res. 2020;30(3):269-71. https://doi.org/10.1038/s41422-020-0282-0.
15. Holshue ML, DeBolt C, Lindquist S, Lofy KH, Wiesman J, Bruce $\mathrm{H}$, et al. First case of 2019 Novel Coronavirus in the United States. N Engl J Med. 2020;382(10):929-36. https://doi.org/10.1056/ NEJMoa2001191.

16. Grein J, Ohmagari N, Shin D, Diaz G, Asperges E, Castagna A, et al. Compassionate use of remdesivir for patients with severe Covid19. N Engl J Med. 2020;382(24):2327-36. https://doi.org/10.1056/ NEJMoa2007016.

17. Beigel JH, Tomashek KM, Dodd LE, Mehta AK, Zingman BS, Kalil AC, et al. Remdesivir for the treatment of Covid-19 - final report. N Engl J Med. 2020;383(19):1813-26. https://doi.org/10. 1056/NEJMoa2007764.

18. Wang Y, Zhang D, Du G, Du R, Zhao J, Jin Y, et al. Remdesivir in adults with severe COVID-19: a randomised, double-blind, placebo-controlled, multicentre trial. Lancet. 2020;395(10236):1569-78. https://doi.org/10.1016/S0140-6736(20)31022-9.

19. Spinner CD, Gottlieb RL, Criner GJ, Arribas López JR, Cattelan AM, Soriano Viladomiu A, et al. Effect of Remdesivir vs standard care on clinical status at 11 days in patients with moderate COVID19: A Randomized Clinical Trial. Jama. 2020;324(11):1048-57. https://doi.org/10.1001/jama.2020.16349.

20. Rivera DR, Peters S, Panagiotou OA, Shah DP, Kuderer NM, Hsu CY, et al. Utilization of COVID-19 treatments and clinical outcomes among patients with cancer: a COVID-19 and Cancer Consortium (CCC19) Cohort Study. Cancer Discov. 2020;10(10):1514-27. https://doi.org/10.1158/2159-8290.Cd-20-0941.

21. Lee S, Santarelli A, Caine K, Schritter S, Dietrich T, Ashurst J. Remdesivir for the treatment of severe COVID-19: a community hospital's experience. J Am Osteopath Assoc. 2020;120(12):92633. https://doi.org/10.7556/jaoa.2020.156.

22. Goldman JD, Lye DCB, Hui DS, Marks KM, Bruno R, Montejano $\mathrm{R}$, et al. Remdesivir for 5 or 10 days in patients with severe Covid19. N Engl J Med. 2020;383(19):1827-37. https://doi.org/10.1056/ NEJMoa2015301.

23. Pasquini Z, Montalti R, Temperoni C, Canovari B, Mancini M, Tempesta M, et al. Effectiveness of remdesivir in patients with COVID-19 under mechanical ventilation in an Italian ICU. J Antimicrob Chemother. 2020;75(11):3359-65. https://doi.org/10. 1093/jac/dkaa321.

24. Lapadula G, Bernasconi DP, Bellani G, Soria A, Rona R, Bombino $\mathrm{M}$, et al. Remdesivir use in patients requiring mechanical ventilation due to COVID-19. Open Forum Infect Dis. 2020;7(11): ofaa481. https://doi.org/10.1093/ofid/ofaa481.

25. Pan H, Peto R, Henao-Restrepo AM, Preziosi MP, Sathiyamoorthy V, Abdool Karim Q, et al. Repurposed antiviral drugs for Covid-19 — Interim WHO Solidarity Trial Results. N Engl J Med. 2020;384: 497-511. https://doi.org/10.1056/NEJMoa2023184.

26. Antinori S, Cossu MV, Ridolfo AL, Rech R, Bonazzetti C, Pagani $\mathrm{G}$, et al. Compassionate remdesivir treatment of severe Covid-19 pneumonia in intensive care unit (ICU) and Non-ICU patients: clinical outcome and differences in post-treatment hospitalisation status. Pharmacol Res. 2020;158:104899. https://doi.org/10.1016/j. phrs.2020.104899.

27. Kalil AC, Patterson TF, Mehta AK, Tomashek KM, Wolfe CR, Ghazaryan V, et al. Baricitinib plus remdesivir for hospitalized adults with Covid-19. N Engl J Med. 2020;384:795-807. https:// doi.org/10.1056/NEJMoa2031994.

28. Falcao F, Viegas E, Carmo I, Soares J, Falcao M, Solano M, et al. A prospective, observational study to evaluate adverse drug reactions in patients with COVID-19 treated with remdesivir or hydroxychloroquine: a preliminary report. Eur J Hosp Pharm. 2021: https://doi.org/10.1136/ejhpharm-2020-002613.

29. Piscoya A, Ng-Sueng LF, Parra del Riego A, Cerna-Viacava R, Pasupuleti V, Roman YM, et al. Efficacy and harms of remdesivir for the treatment of COVID-19: a systematic review and meta- 
analysis. PLoS One. 2020;15(12):e0243705. https://doi.org/10. 1371/journal.pone.0243705.

30. Verdugo-Paiva F, Acuña MP, Solá I, Rada G. Remdesivir for the treatment of COVID-19: a living systematic review. 2020;20(11): e8080. https://doi.org/10.5867/medwave.2020.11.8080.

31. Siemieniuk RA, Bartoszko JJ, Ge L, Zeraatkar D, Izcovich A, Kum E, et al. Drug treatments for covid-19: living systematic review and network meta-analysis. Bmj. 2020;370:m2980. https://doi.org/10. 1136/bmj.m2980.
32. Elsawah HK, Elsokary MA, Abdallah MS, ElShafie AH. Efficacy and safety of remdesivir in hospitalized Covid-19 patients: systematic review and meta-analysis including network meta-analysis. Rev Med Virol. 2021;31(4):e2187 https://doi.org/10.1002/rmv. 2187.

Publisher's Note Springer Nature remains neutral with regard to jurisdictional claims in published maps and institutional affiliations. 